\title{
External carotid occlusive disease as a cause of facial pain
}

\author{
Y. HERISHANU ${ }^{1}$, P. BENDHEIM ${ }^{2}$, AND M. DOLBERG \\ From the Neurology Unit and Department of Radiology, \\ Shaare Zedek General Hospital, Jerusalem, Israel
}

SYNOPSIS A 47 year old man suffered an acute left hemiparesis after several weeks of right-sided facial pain. Right carotid angiography revealed internal carotid artery thrombosis and severe occlusion of external carotid branches supplying facial structures. An ischaemic aetiology for the facial pain is suggested.

The differential diagnosis of facial pain has been extensively reviewed in the literature (Friedman, 1966; Hurwitz, 1968; DeLeon, 1968; Burton, 1969; Foster, 1969). Among the common entities are odontogenic disease, trigeminal and glossopharyngeal neuralgias, migrainous facial pain, post-herpetic neuralgia, various neoplasms, giant cell arteritis, cardiovascular facial pain, and the pain associated with psychoneurotic conditions. Other causes are recognized but, nevertheless, there remains a significant number of cases for which no cause is found. These undiagnosed cases are generally categorized as atypical or idiopathic facial pain. The following case history supports the concept that arterial insufficiency in the distribution of the external carotid artery can produce an ischaemic neuralgia. A similar pathogenesis of facial pain may be the mechanism in other cases classified as atypical.

\section{CASE HISTORY}

A 47 year old man was admitted to the emergency room where the diagnosis of an acute left hemiparesis was made. In the four weeks before the onset of his hemiparesis, the patient complained of severe pain in the teeth, ear, and face on the right side. Three days before admission he consulted an oral surgeon. No dental disease was found to explain the

Address for correspondence: Dr Y. Herishanu, Neurology Unit, Shaare Zedek General Hospital, P. O. B. 293, Israel.

2 Present address: University of Arizona, College of Medicine, Tucson, Arizona, U.S.A. pain, but the last right maxillary molar was extracted without relief. Two days before hospitalization he experienced dizziness. Other past history was noncontributory, but the family history revealed hypertension and coronary artery disease in the patient's father.

On admission to the medical ward his blood pressure was $150 / 100 \mathrm{mmHg}$, heart rate was 92 per minute and regular. The patient was restless but mental status and speech were normal. Funduscopic examination revealed a grade 1 arteriosclerotic retinopathy. A left hemiparesis and left, upper motor neurone facial paresis were evident. The sensory examination was normal. Carotid artery palpation elicited no tenderness and no bruits were detected on carotid auscultation.

The electrocardiogram showed evidence of left ventricular hypertrophy. Radiographs of the chest and skull were normal. An electroencephalogram demonstrated slowing of electrical activity in the right frontotemporal region.

Laboratory studies included haemoglobin $14.5 \mathrm{~g} /$ $100 \mathrm{ml}$, haematocrit $43 \%$, white blood cell count 9,000 per $\mathrm{mm}^{3}$, and a normal erythrocyte sedimentation rate. Blood glucose, urea, protein, enzymes, and electrolytes were within normal limits. No elevation was found of blood cholesterol, triglycerides, or total lipids. A serological test for syphilis was negative, as was an anti-nuclear factor determination and an LE cell preparation.

A right carotid arteriogram was performed three days after admission (Figure). The internal carotid artery was completely blocked. The facial artery was almost totally occluded near its origin from the external carotid, while the internal maxillary artery 


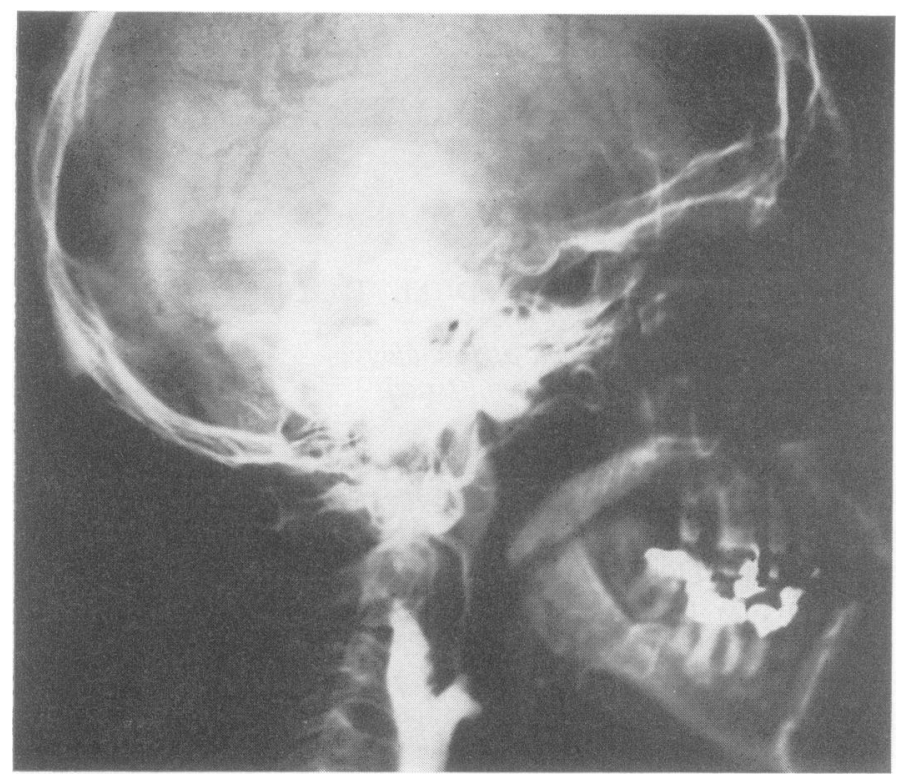

FIGURE Right common carotid arteriogram. Complete internal carotid occlusion and severe stenosis of the external carotid and its branches are evident.

showed stenosis. Right anterior and middle cerebral arteries were seen to fill via external carotid collateral branches. A nearly complete recovery from the hemiparesis was evident on a follow-up examination two months later.

\section{DISCUSSION}

Before the angiographic studies for left hemiparesis no cause was discovered for his rightsided facial pain. The classification of his condition as being atypical or idiopathic facial pain was made after medical and dental examination failed to identify one of the usual causes. However, the radiographic evidence of an occlusive vascular process in the major branches of the right external carotid artery enabled us to propose that ischaemia affecting peripheral sensory fibres innervating facial structures was the mechanism producing our patient's pain.

The severe nature of ischaemic pain is recognized in conditions such as angina pectoris and thromboembolic disease of the extremities. In the literature reviewed, however, occlusive vascular disease producing ischaemic facial pain is not a prominent consideration in aetiology. Friedman et al. (1960) include facial pain of vascular origin in their category of atypical pains. They mention that the mechanism responsible is not well understood. Norman (1970) identifies intermittent claudication of the masticatory muscles due to arteritis of the external carotid artery branches as a cause of some atypical pains.

The pathogenesis of pain in occlusive vascular disease can be an ischaemia affecting the nerves themselves (Stead, 1970). In patients with facial pain who are at risk to the development of occlusive vascular disease, due to pre-existing diabetes, hypertension, atherosclerosis, or other predisposing conditions, the possibility exists that an ischaemic neuritis in the external carotid distribution may be the responsible pathology.

Arteriography in our case demonstrated occlusive external carotid artery disease. Since dental disease, trigeminal neuralgia, and other common causes had been ruled out, we postulated an ischaemic mechanism. A similar pathogenesis might be responsible in other cases of atypical facial pain. Diagnosis of occlusive external carotid vascular disease in at-risk patients with facial pain, and the awareness that similar processes may coexist in the common and internal carotid arteries, may be of prognostic and therapeutic value. 


\section{REFERENCES}

Burton, R. C. (1969). The problem of facial pain. Journal of the American Dental Association, 79, 93-101.

DeLeon, E. L. (1968). Facial pain of non-odontogenic origin. Journal of Oral Medicine, 23, 119-131.

Foster, J. B. (1969). Facial pain. British Medical Journal, 4, 667-669.

Friedman, A. P. (1966). Differential diagnosis of facial pain. Dental Clinics of North America, 545-551.
Friedman, A. P., Carton, C. A., and Hirano, A. (1960). Cyclical head and face pain: its diagnosis and treatment. Archives of Neurology (Chic.), 2, 1-11.

Hurwitz, L. J. (1968). Facial pain of non-dental origin. British Dental Journal, 124, 167-171.

Norman, J. E. de B. (1970). Facial pain and vascular disease. British Journal of Oral Surgery, 8, 138-144.

Stead, E. A., Jr (1970). Pain in the extremities. In Harrison's Principles of Internal Medicine, 6th edn, pp. 78-81. McGraw-Hill: New York. 